\title{
Clinical and Topographical Corneal Changes after Keraring Implantation in Keratoconus Patients
}

\author{
Mirzaei Mohamad ${ }^{1}$, Najafi Amin $^{2 *}$, Shahraki Kurosh ${ }^{3}$, Taheri Nazli ${ }^{4}$, Mirzaei Samira ${ }^{5}$
}

1. Assistant professor of ophthalmology Tabriz university of medical sciences

2. Resident of ophthalmology Tabriz university of medical sciences

3. Resident of ophthalmology Iran university of medical sciences

4. Fellowship of anterior segment Tabriz university of medical sciences

5. General practitioner

\begin{abstract}
Introduction: Keratoconus is a progressive, non-inflammatory, bilateral, ecstatic disease of the cornea that is characterized by corneal steepening which progresses to irregular conical shape, with subsequent irregular astigmatism and decreased visual acuity. In early stages of disease, spectacles or RGP lenses are the main treatment. At advanced stages, when these treatment options fail to correct visual acuity, surgical procedures such as penetrating keratoplasty or lamellar keratoplasty are required. METHODS: Keraring segments were placed in 30 eyes of 21 keratoconus patients. The mean follow-up time was 6.2 months. All patients underwent a complete ocular examination and corneal topography which was obtained by Pentacam (Oculus Pentacam ${ }^{\circledR}$, USA). Improvement in uncorrected visual acuity (UCVA), best spectacle corrected visual acuity, refraction and topographic changes including keratometry, maximum anterior and posterior elevation, central corneal thickness and thinnest point thickness and location were evaluated.

RESULTS: No intraoperative or postoperative complications occurred in any of the patients. ICRS implantation significantly improved UCVA $(P=0.005)$ and BSCVA $(P=0.000)$. Mean Spherical equivalent significantly reduced from $-6.66 \pm 3.52$ to $-4.42 \pm 3.20(p=0.000)$. A clinically significant reduction in mean keratometry $(p=0.000)$, Max Ant Elevation ( $P=0.024)$ and Max Post Elevation $(P=0.002)$ were recorded. No clinically significant changes in central corneal thickness, thinnest point thickness and thinnest point location were observed.

Conclusion: With mean follow-up of 6.2 months, Keraring segments implantation caused significant clinical improvement in UCVA, BCVA Refractive Error and Keratometric findings of the most keratoconic patients.
\end{abstract}

Keywords: keratoconus; ICRS; Keraring

\section{Corresponding author:}

Najafi, Amin

Resident of ophthalmology Tabriz university of medical sciences

Receive date: 2014-08-16| Accept date: 2014-08-29| Publish date: 2014-09-19

DOI: 10.7575/aiac.abcmed.15.03.01.10 


\section{Introduction}

Keratoconus is a progressive, noninflammatory, bilateral, ecstatic disease of the cornea that is characterized by corneal steepening which progresses to irregular conical shape, with subsequent irregular astigmatism and decreased visual acuity. At early stages of disease, spectacles or RGP lenses are the main treatment. At advanced stages, when these treatment options fail to correct visual acuity, surgical procedures such as penetrating keratoplasty (PK) or lamellar keratoplasty are required (1-4).

Different alternative methods for keratoconus treatment are introduced and reported in the literature, such as penetrating keratoplasty, Phakic IOLs, photorefractive keratectomy, CXL, laser in situ keratomileusis, and lamellar keratoplasty (5-6).

ICRS firs were developed for correction of low myopia but later studies showed that these rings are effective for correction of myopia and irregular astigmatism secondary to keratoconus $(1,7-9)$.

Keraring is a type of ICRS which acts by flattening the central cornea and thus reducing existing myopia and astigmatism by morphological irregularities correction. The first Keraring design, $\mathrm{SI}-5$, had triangular cross section and was available as $90^{\circ}, 120^{\circ}, 160^{\circ}$, $210^{\circ}$ and $240^{\circ}$ segments made of PMMA with an optical diameter of $5 \mathrm{~mm}$ and base widths of $6 \mathrm{~mm}$. They came in variable thickness $(0.15-$ $0.35 \mathrm{~mm}$ ) in $0.05 \mathrm{~mm}$ steps. SI-6 has recently been designed with a 6-mm optical zone and $800-\mu \mathrm{m}$ base width $(10,11)$.

The purpose of this study was to evaluate the visual acuity, refraction, and corneal tomographic and pachymetric changes after Keraring implantation in keratoconic eyes.

\section{Methods}

Ethical Committee Approval at Tabriz University of medical science was obtained for this study. After giving necessary information about the study, written informed consent was obtained from the patients. In this prospective Before-After study, twenty-one patients (30 eyes) were included. Patients were older than 18 years, contact lens intolerant, and of a follow-up of at least 6 months. A complete ophthalmologic examination was performed before operation to exclude any other ocular diseases. Patients were excluded if they had any of the following criteria: Central corneal opacity, history of HSV keratitis, history of autoimmune diseases, systemic collagen vascular disease, central corneal thickness less than 400 micrometer and K-max of more than 60 diopters. Slit lamp examination, UCVA, BCVA, manifest refraction and corneal topography with Pentacam (Oculus Pentacam ${ }^{\circledR}$, USA) were evaluated before and at least 6 month after surgery and results were compared. Operation was performed under local anesthesia.Size, thickness and ring numbers needed for every patient was determined according to the refraction, corneal topography and the Keraring nomogram provided by the manufacturer. At first the geometric center of the cornea was determined with a surgical Caliper. Then, with a circular marker the central $5 \mathrm{~mm}$ optical zone was marked and the incision site in the steep meridian determined. An $80 \%$ thickness corneal radial incision was made with a calibrated diamond knife. Then, a hook used to create a pocket on either side of incision. At the next step, the clockwise and counterclockwise dissector was used to create tunnels in the corneal stroma to implant ring segments. Finally, the incision site was repaired with a 100 nylon suture. Postoperatively, all eyes received antibiotic eye drops four times daily for one week and tapering dose of 
corticosteroid eye drops for 3 weeks. The sutures were removed after 6 weeks. Visual acuity, refraction and corneal topographic and pachymetric changes were evaluated 6 months after surgery. Kolmogorov-Smirnov test was used to check the normality of all data. If parametric analysis was possible, Paired t-test was used for comparisons between preoperative and postoperative examinations. If parametric analysis was not possible, the Wilcoxon test was performed. $\mathrm{P}<0.05$ was considered to be significant in all case.

\begin{tabular}{lccc} 
parameter & preoperation & postoperation & P value \\
\hline UCVA & $0.81 \pm 0.22$ & $0.63 \pm 0.29$ & 0.005 \\
BCVA & $0.42 \pm 0.20$ & $0.20 \pm 0.17$ & 0.000 \\
SRE & $-4.21 \pm 3.45$ & $-2.69 \pm 2.99$ & 0.000 \\
CRE & $4.86 \pm 2.31$ & $2.88 \pm 1.98$ & 0.000 \\
SE & $-6.66 \pm 3.52$ & $-4.42 \pm 3.20$ & 0.000 \\
Mean KR & $49.57 \pm 3.98$ & $47.03 \pm 3.32$ & 0.000 \\
Max Ant Elevation & $35.03 \pm 13.93$ & $29.63 \pm 17.30$ & 0.024 \\
Max post Elevation & $71.13 \pm 22.76$ & $60.06 \pm 20.94$ & 0.002 \\
CCT & $466.83 \pm 29.19$ & $469.06 \pm 30.10$ & 0.505 \\
TP & $448.86 \pm 32.26$ & $451.90 \pm 41.52$ & 0.538 \\
T.PL dislocation & $0.71 \pm 0.20$ & $0.65 \pm 0.25$ & 0.184
\end{tabular}

\section{Results}

Kerarings were successfully implanted in all eyes. The mean follow up time was $6.2 \pm 0.6$ (67.5) months. Mean age of patients was $27.10 \pm 5.10$ years. 12 patients $(57.14 \%)$ were female and 9 patients (42.86\%) were male. The mean UCVA (Mean LogMAR) significantly improved after Keraring implantation from $0.81 \pm 0.22$ to $0.63 \pm 0.29$ at the last follow-up.

Table 1: Comparision of pre and post operation results

UCVA; uncorrected visual acuity, BCVA; best corrected visual acuity, SRE; spherical refractive error, CRE; cylindrical refractive error, $\mathrm{SE}$; spherical equivalent, KR; keratometric readings, CCT; central corneal thickness, TP; thinnest point, TPL; thinnest point location

$(P=0.005)$. Best corrected visual acuity (BCVA) also significantly changed from $0.42 \pm 0.20$ (mean LogMAR) to $0.20 \pm 0.17(\mathrm{P}=0.000)$. Mean preoperative spherical, cylindrical refractive error and spherical equivalent were $-4.21 \pm 2.45$, $-4.86 \pm 2.31,-6.66 \pm 3.52$, respectively (Table-1). A significant reduction in spherical $R E$, cylindrical
RE and SE to $-2.69 \pm 1.99,-2.88 \pm 1.98,-4.42 \pm 3.20$ respectively, was reported ( $P=0.000$ for all). $A$ significant reduction in keratometric values was found at Post operative examination. Mean preoperative keratometry significantly changed from $49.57 \pm 3.98$ to $47.03 \pm 3.32$ diopters $(P=0.000)$. Mean values of Max Ant Elevation 
and Max Post elevation were $35.03 \pm 13.93$ and $71.13 \pm 22.76$, respectively which reduced significantly to $29.63 \pm 17.30$ and $60.06 \pm 20.94$ $(\mathrm{P}=0.024,0.002)$. Mean preoperative Central Corneal Thickness (CCT) was $466.83 \pm 29.19$ that changed to $469.06 \pm 30.10$ which was not statistically significant $(P=0.505)$. Thinnest point location changes along $X$ and $Y$ axis were also evaluated by calculation of the line length that drawn from the center of the cornea to thinnest point location. The analysis results showed that no significant change was observed in the line length between before and after surgery condition which indicating of thinnest point location changes $(P=0.184)$.

\section{Discussion}

One important benefit of treating keratoconus with ICRS is to delay the corneal graft with subsequent complication or eliminate this need. ICRS does not eliminate the corneal disease but decreases the associated corneal abnormality and improves visual acuity in affected patients to satisfactory levels. An important advantage of this method is the removability of ICRS $(5,6)$.

Colin and associates (12) first showed that Intacs can decrease the corneal steepening and astigmatism in keratoconus patients. They later concluded that Intacs is an effective method for treating keratoconus patients which are contact lens intolerant and have clear cornea. However, it should be taken into consideration that sometimes corneal response to these segments in keratoconic patients is unpredictable because of the variable responses of the eyes $(12,13)$.

Various factors such as the location of the cone, stage of the keratoconus and the biomechanics of the eye can influence the response to treatment (11).

In this study, also significant corneal flattening changes (mean change of $2.21 \mathrm{D}$ in mean keratometry values) was observed after KeraRing implantation. These results are similar to the findings reported in previous studies. It seems that the main reason for the change in refraction and the increase in UCVA and BCVA is this keratometric changes. As reported by previous studies, keratometric change after ring implantation was dependent on several preoperative factors such as keratometry, BSCVA and refraction. Nomograms, which take into account the cones morphology for ring selection, were firstly presented by Colin (14).

Torquetti et al. (15) combined Colin method with the distribution of ectasia based on the percentage of cone on either side of the midline.

In another study, Ganesh et al. (11) showed that planning of the ring placement based on the morphology of the cone in addition to the patient's refraction was associated with proper outcomes.

In this study, also size, thickness and ring numbers needed for every patient are determined according to refraction, corneal topography and the nomogram provided by the manufacturer that may yield in better outcomes.

Pinero et al. (16) reported that ICRS implantation with both mechanical and femtosecond laser-assisted procedures have similar visual and refractive results. In this study, we used mechanical procedures but it seems that the use of the femtosecond laser makes the corneal tunnel creation easier, faster and more accurate.

Similar to the results reported by Sogutlu et al.(17), Max Anterior and Posterior elevation of the cornea reduced significantly. Although the anterior corneal surface optically is more important than the posterior corneal surface,(18) posterior corneal surface optical contribution can become relevant in keratoconus, especially when associated with a high curvature. (16)

The results of our study are similar to the results of ICRS implantation in low myopic patients,(19-21) keratoconus, (22-27) and post- 
LASIK ectasia (28-31) while refraction and visual acuity were stable after the sixth month. No intra- or postoperative complications occurred in this study.

It seems that the major changes in visual acuity, refraction, and topographic findings occur during the first 6 months. Although the visual acuity and refraction improve after ICRS implantation, chronic corneal stroma remodeling secondary to keratoconus progression occurs and may influence long term efficacy. Present study also showed that there is no significant correlation (only a weak correlation) between Max Ant Elevation and Max Post elevation changes rate after surgery. Also a similar correlation was determined between mean keratometry values with both Ant and Post elevation changes. Similar to Saelens et al.(32) reports, in this study also central corneal thickness changes after ICRS implantation were insignificant. According to this study, thinnest point location changes along the $X$ and $Y$ axis were not significant; however, to the best of our knowledge, there are no reports evaluating the effect of implantation of ICRS on thinnest point location , so further studies with additional cases are needed to judge about this parameter.

The possible limitations that are apparent in this study are the small sample of studied eyes and limited post operation follow up period.

\section{Conclusion}

Keraring intra-stromal corneal ring segments implantation are an effective and safe procedure for keratoconus patients and significantly improves the corneal tomographic properties with subsequent correction in UCVA and BCVA.

\section{References}

1. Espandar, L. and J. Meyer (2010). "Keratoconus: overview and update on treatment." Middle East Afr J Ophthalmol 17(1): 15-20.

2. de Freitas Santos Paranhos, J., et al. (2011). "Visual perception changes and optical stability after intracorneal ring segment implantation: comparison between 3 months and 1 year after surgery." Clin Ophthalmol 5: 1057-1062.

3. Hashemi, H., et al. (2013). "Efficacy of intacs intrastromal corneal ring segment relative to depth of insertion evaluated with anterior segment optical coherence tomography. " Middle East Afr J Ophthalmol 20(3): 234-238.

4. Vazirani, J. and S. Basu (2013). "Keratoconus: current perspectives." Clin Ophthalmol 7: 2019-2030.

5. Siganos, C. S., et al. (2003). "Management of keratoconus with Intacs." Am J Ophthalmol 135(1): 64-70.

6. Colin, J., et al. (2001). "INTACS inserts for treating keratoconus: one-year results." Ophthalmology 108(8): 14091414.

7. Pinero, D. P., et al. (2010). "Modeling the intracorneal ring segment effect in keratoconus using refractive, keratometric, and corneal aberrometric data." Invest Ophthalmol Vis Sci 51(11): 5583-5591.

8. .Kymionis, G. D., et al. (2007). "Long-term follow-up of Intacs in keratoconus." Am J Ophthalmol 143(2): $236-244$.

9. Shabayek, M. H. and J. L. Alio (2007). "Intrastromal corneal ring segment implantation by femtosecond laser for keratoconus correction." Ophthalmology 114(9): 1643-1652.

10. Ertan, A. and J. Colin (2007). "Intracorneal rings for keratoconus and keratectasia." J Cataract Refract Surg 33(7): 1303-1314.

11. Ganesh, S., et al. (2013). "Intrastromal corneal ring segments for management of keratoconus." Indian J Ophthalmol 61(8): 451-455.

12. Colin, J., et al. (2000). "Correcting keratoconus with intracorneal rings." J Cataract Refract Surg 26(8): 1117-1122.

13. Tunc Z, Deveci N, Sener B, Bahcecioglu H. Anneaux intracornee'ns (Intacs) pour le traitement de l'astigmatisme asyme’trique du ke'ratoco ^ ne: recul de plus de deux ans. J Fr Ophtalmol 2003; 26:824-830.

14. Colin J. European clinical evaluation: Use of Intacs for the treatment of keratoconus. J Cataract Refract Surg 2006;32:747-55. 
15. Torquetti L, Berbel RF, Ferrara P. Long-term follow-up of intrastromal corneal ring segments in keratoconus. J Cataract Refract Surg 2009;35:1768-73.

16. Pinero, D. P., et al. (2009). "Refractive and aberrometric outcomes of intracorneal ring segments for keratoconus: mechanical versus femtosecond-assisted procedures." Ophthalmology 116(9): 1675-1687.

17. Sogutlu, E., et al. (2012). "Elevation changes of central posterior corneal surface after intracorneal ring segment implantation in keratoconus." Cornea 31(4): 387-395.

18. Turner T. What corneal topography can tell you about corneal shape. In: MacRae S, Krueger R, Applegate RA, eds. Customized Corneal Ablation: The Quest for Supervision. Thorofare, NJ: Slack Inc; 2001:221-227.

19. Rapuano CJ, Sugar A, Koch DD, et al. Intrastromal corneal ring segments for low myopia: a report by the American Academy of Ophthalmology. Ophthalmology 2001;108:1922- 1928.

20. Schanzlin Dj, Abbot RI, Asbell PA, et al. Two-year outcomes of intrastromal corneal ring segments for the correction of myopia. Ophthalmology 2001;108:1688 -1694.

21. Schanzlin DJ, Asbell PA, Burris TE, Durrie DS. The intrastromal corneal ring segments. Phase II results for the correction of myopia. Ophthalmology 1997;104:1067-1078.

22. Guell JL. Are intracorneal rings still useful in refractive surgery? Curr Opin Ophthalmol 2005;16:260 -265.

23. Siganos CS, Kymionis GD, Kartakis N, et al. Management of keratoconus with Intacs. Am J Ophthalmol 2003;135:64 $-70$.

24. Boxer Wachler BS, Christie JP, Chandra NS, et al. Intacs for keratoconus. Ophthalmology 2003;110:1031-40.

25. Sharma M, Boxer Wachler BS. Comparison of single-segment and double-segment Intacs for keratoconus and postLASIK ectasia. Am J Ophthalmol 2006;141:891- 895.

26. Alio, J. L., et al. (2006). "Analysis of results related to good and bad outcomes of Intacs implantation for keratoconus correction." J Cataract Refract Surg 32(5): 756-761.

27. Alio JL, Shabayek MH, Artola A. Intracorneal ring segments for keratoconus correction: long-term follow-up. J Cataract

Refract Surg 2006;32:978-985

28. Kymionis GD, Siganos CS, Kounis G, et al. Management of post-LASIK corneal ectasia with Intacs inserts: one-year results. Arch Ophthalmol 2003;121:322-326.

29. Kymionis GD, Tsiklis NS, Pallikaris Al, et al. Long-term follow-up of Intacs for post-LASIK corneal ectasia. Ophthalmology 2006;113(11) Forthcoming.

30. Siganos CS, Kymionis GD, Astyrakakis N, Pallikaris IG. Management of corneal ectasia after laser in situ keratomileusis with INTACS. J Refract Surg 2002;18:43- 46.

31. Alio J, Salem T, Artola A, Osman A. Intracorneal rings to correct corneal ectasia after laser in situ keratomileusis.

32. Saelens, I. E., et al. (2011). "Refractive, topographic, and visual outcomes of same-day corneal cross-linking with Ferrara intracorneal ring segments in patients with progressive keratoconus." Cornea 30(12): 1406-1408. 